\title{
Stereotactic Radiosurgery and Stereotactic Radiotherapy for Brain Tumors
}

\section{Enis Tinjak*, Velda Smajlbegović, Nusret Salkica and Teufik Orahovac}

Clinical Center University of Sarajevo, Sarajevo, Bosnia and Herzegovina

*Corresponding Author: Enis Tinjak, Medical Radiology Technologist, RTT, Clinical

Center University of Sarajevo, Sarajevo, Bosnia and Herzegovina.
Received: June 28, 2021

Published: August 07, 2021

(C) All rights are reserved by Enis Tinjak., et al.

\begin{abstract}
Stereotactic radiosurgery (SRS) and fractionated stereotactic radiotherapy (FSRT) have become important treatment modalities for brain tumors. A technique that delivers a high dose of radiation, with effective use in patients with life-limiting diseases. Due to its specificity, the precision and direct visualization of the target during the delivery of the treatment is very important. The aim of this research article is to provide the safety and long-term efficacy of SRS and SRT. This research article, also, provides accuracy of the SRS treatment performed on linear accelerator and use of the trUpoint ARCH SRS system for patient imobilization.
\end{abstract}

Keywords: Stereotactic Radiosurgery; Radiotherapy; Brain Metastases and Tumors; Linear Accelerator

\section{Introduction}

Stereotactic radiosurgery (SRS) is an effective technique for irradiating brain tumors, in which a high dose of radiation is delivered in a single fraction of treatment, to a small intracranial volume while saving normal healthy structures.

Stereotactic radiotherapy (SRT) is a non-invasive radiotherapy technique that delivers a high dose of radiation in multiple treatment fractions ( $2-5)$ to a target volume known as fractionated stereotactic radiotherapy (FSRT) [1].

The goal is to capture the target volume at a high radiation dose and use a steep dose gradient to spare the surrounding normal tissue.

Hypofractionated radiotherapy allows the delivery of high doses of radiation per fraction while minimizing adverse effects, all while maintaining good local tumor control. SRT is increasingly becoming the preferred treatment for intracranial metastatic le- sions, as well as brain tumors up to $3 \mathrm{~cm}$ in size, not only because of its efficacy in providing good local control, but also because of its limited long-term toxicity, especially in terms of neurocognitive function [2].

There are three types of technology to deliver radiation during stereotactic radiosurgery in the brain and other parts of the body: Linear accelerator (LINAC) machines use X-rays (photons) to treat cancerous and noncancerous abnormalities in the brain and other parts of the body. These machines can perform stereotactic radiosurgery (SRS) in a single session or over three to five sessions for larger tumors, which is called fractionated stereotactic radiotherapy.

Gamma Knife machines use 192 or 201 small beams of gamma rays to target and treat cancerous and noncancerous brain abnormalities.

Proton beam therapy (charged particle radiosurgery) is the newest type of stereotactic radiosurgery and can treat brain cancers 
in a single session using stereotactic radiosurgery, or it can use fractionated stereotactic radiotherapy to treat body tumors over several sessions $[3,4]$.

The brain imaging process, as well as the treatment of tumors, whether benign or malignant, is very important for patients with these diseases. Several studies have been carried out that used radioactive materials such as technetium-99m [5-8], iodine [9] and others, according to [10-12].

The patients involved in this trial were treated in a two different treatment regimens, single-dose radiosurgery (SRS) and fractionated stereotactic radiotherapy (FSRT).

Most patients treated with the stereotactic radiotherapy technique are patients with intracranial metastases as well as a few patients with benign tumors.

\section{Stereotactic radiotherapy for brain tumors}

All patients in whom stereotactic treatment of brain tumors was indicated were irradiated with the Volumetric Modulated Arc Therapy (VMAT), RapidArc, on the linear accelerator TrueBeam, Varian (Palo Alto, CA) with a photon energy of 6MV flattening filter free (FFF). Image-guided radiotherapy (IGRT) applied to verify patient position, alignment and geometric accuracy is performed using On Board Imaging (OBI) kV imaging systems, Cone Beam computed tomography (CBCT) and Kv-kv pair imaging.

In this research, a retrospective analysis from 2019 to 2021 included 35 patients treated with stereotactic radiotherapy.

Most of the patients were female 20, while there were 15 male patients. The youngest patient was 14 years old while the oldest one was 81 years old, and the mean age of all patients was 56.5 years.

Of the total number of patients (35), most of them were diagnosed with a metastatic brain tumor (28) for which stereotactic treatment was indicated. In addition to metastatic changes, patients with vestibular Schwannoma (4), trigeminal neuralgia (1), recurrent meningioma (1) and cerebral pinealocyte (1) were treated with the stereotactic technique (Chart 1 ).

Of the total number of patients (pt) with metastatic changes in the brain (28pt), the most common is a single-fraction treatment

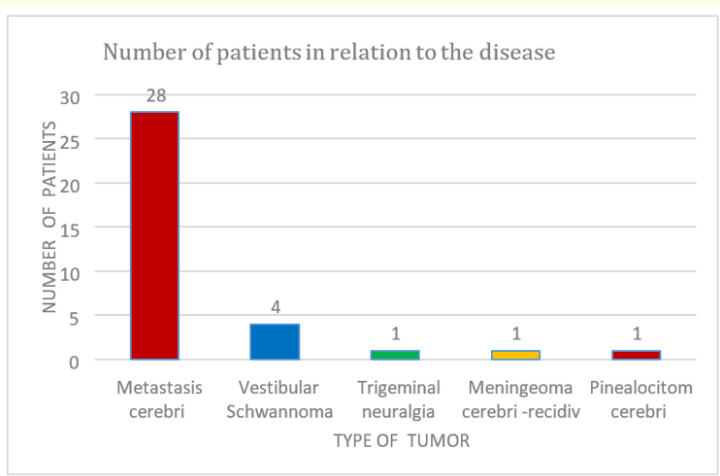

Chart 1: Shows the number of patients in relation to the type of tumor treated.

regimen (15pt) with the most commonly delivered dose of $18 \mathrm{~Gy}$, a fractionated regimen in 5 fractions (7pt) with a dose of $20 \mathrm{~Gy}$, and a fractionated regimen in 3 fractions (6pt) with the most commonly delivered dose of 21 Gy (Chart 2).

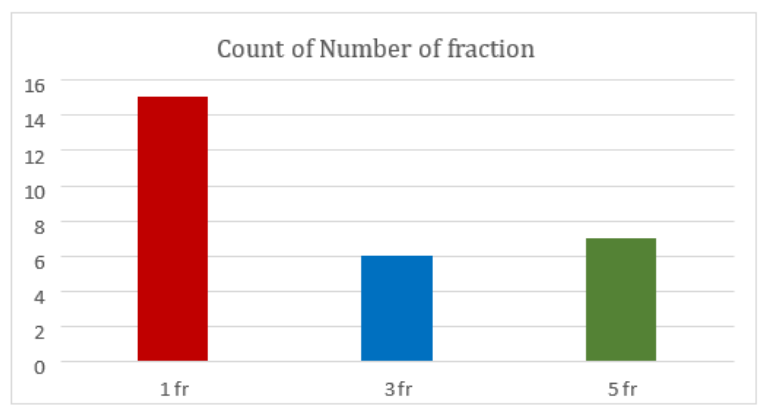

Chart 2: The most frequently used fractionation regimen in relation to the number of patients.

The most of the patients treated for brain metastases, received a dose of 18Gy in a single-fraction regimen of planned treatment dose, (Chart 3).

In addition to the treatment of metastatic brain lesions, table 1 , shows the fractionation regimens and total dose to other represented brain tumors treated with the stereotactic radiotherapy technique. 


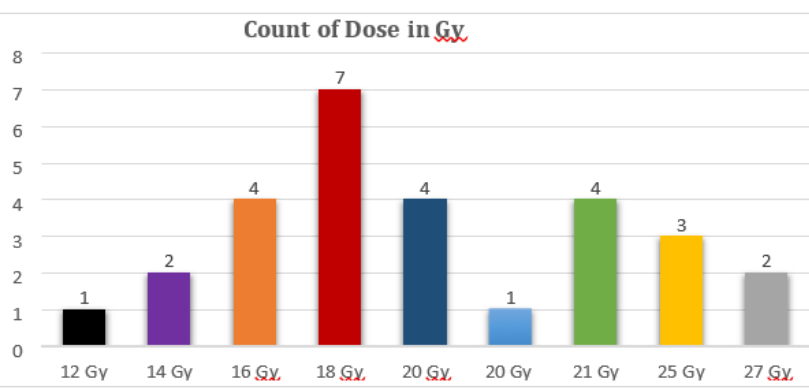

Chart 3: Shows the prescribed radiation doses in relation to the number of patients for brain metastases.

\begin{tabular}{|l|c|c|c|c|}
\hline $\begin{array}{c}\text { Region of the } \\
\text { treatmen }\end{array}$ & $\begin{array}{c}\text { Vestibular } \\
\text { Schwan- } \\
\text { noma }\end{array}$ & $\begin{array}{c}\text { Trigeminal } \\
\text { neuralgia }\end{array}$ & $\begin{array}{c}\text { Recurrent } \\
\text { menin- } \\
\text { gioma }\end{array}$ & $\begin{array}{c}\text { Cerebral } \\
\text { pinealocyte }\end{array}$ \\
\hline $\begin{array}{l}\text { Number of the } \\
\text { patients }\end{array}$ & 4 & 1 & 1 & 1 \\
\hline $\begin{array}{l}\text { Number of } \\
\text { fractions/dose }\end{array}$ & $\begin{array}{c}1 \mathrm{pt} \\
13 \mathrm{~Gy} / 1 \mathrm{fr} \\
14 \mathrm{pt}\end{array}$ & $12 \mathrm{~Gy} / 1 \mathrm{fr}$ & $25 \mathrm{~Gy} / 5 \mathrm{fr}$ & $25 \mathrm{~Gy} / 5 \mathrm{fr}$ \\
& $\begin{array}{c}\text { 2pt } \\
18 \mathrm{~Gy} / 3 \mathrm{fr}\end{array}$ & & & \\
\hline
\end{tabular}

Table 1: Radiation dose and fractionation for other brain tumors in SRT technique.

\section{Immobilization of the patient for SRS treatment}

Positioning and immobilization of the patients for SRS treatment is performed using the trUpoint ARCH system, CIVCO radiotherapy (Figure 1 and 2).

\section{Treatment planning}

Treatment planning for SRS treatment is performed using the Eclipse planning system, Varian. Defining and contouring the target volume and organs at risk is done on the basis of imaging CT simulator for planning (Canon Aquillion LB) and fusion of magnetic resonance imaging (MRI). The verification and QA of the radiotherapy plan on the linear accelerator is performed using a Matrixx

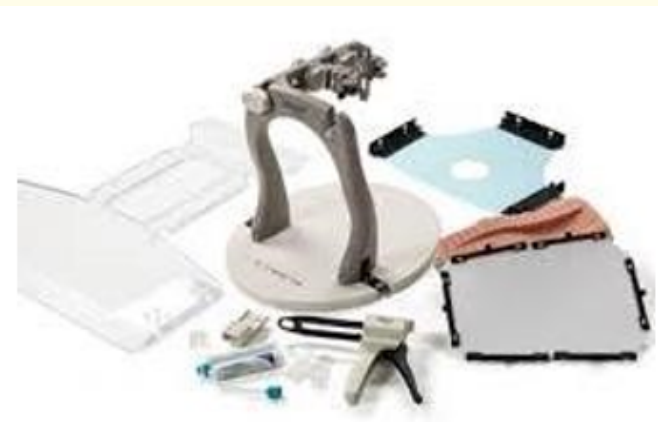

Figure 1: trUpoint ARCH system for SRS.

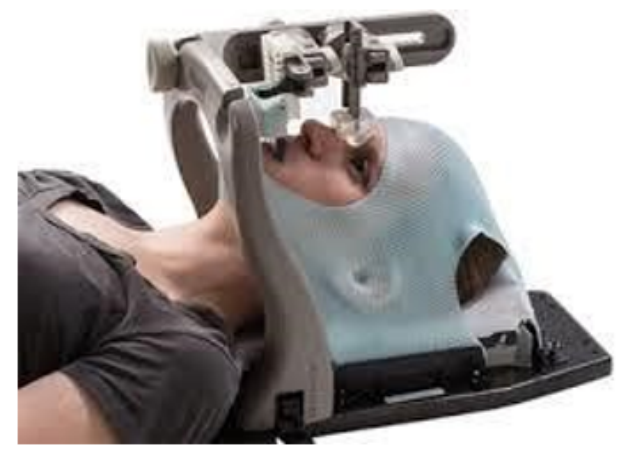

Figure 2: Positioning and immbilization of the patient using the trUpoint ARCH system for SRS treatment.

Evolution phantom (IBA Dosimetry) where the deviations must not exceed $2 \mathrm{~mm}$ and $2 \%$, gamma pass.

\section{IGRT for stereotactic treatment}

The procedure of verification of the patient's position and geometric accuracy during the patient's treatment is performed on the basis of CBCT images.

The patient is positioned in a completely identical position as on the CT simulator following the setup sheet and reference markers on the patient mask. 
According to the plan and defined displacements, a delta couch shift is performed, followed by a CBCT verification (Figure 3 ).

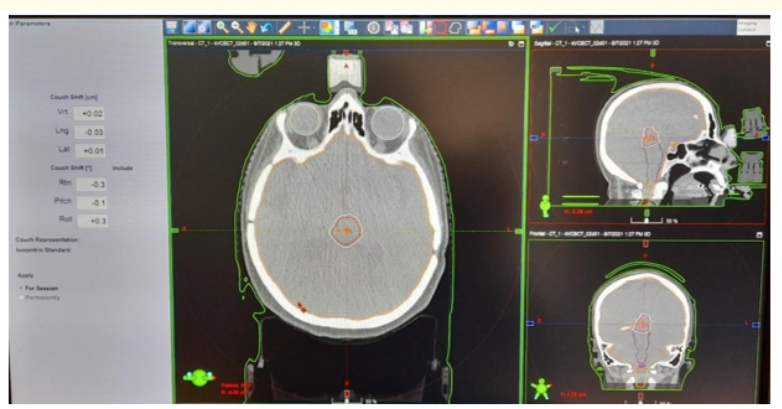

Figure 3: Pretreatment verification of patient position and target using CBCT.

Geometric deviations in translational movements up to $2 \mathrm{~mm}$, as well as pitch and roll up to $2^{0}$, can be are aligned with the patient's treatment table, after satisfactory online verification of the target volume. Matching of images are performed based on bone anatomical structures and online visualization and verification of the target volume. Auto matching is limited within $2 \mathrm{~mm}$ according to the target volume on CBCT images reconstructed per $1 \mathrm{~mm}$ thickness. In case of larger deviations from the above, the patient's position is re-checked and corrected, in consultation with a radiation oncologist. Prior to the delivery of the prescribed dose, the patient is imaged at each table rotation according to the position in the plan. A control CBCT is done at the end of treatment to check for possible intrafractional movements, given the patient's general condition and cooperation during treatment delivery.

Comparing the pretreatment and posttreatment CBCT images, slight submillimeter deviations were observed (Figure 4).

This way of verifying the position of the patient and the target, helped to establish and prove the validity and accuracy of using the trUpoint ARCH immobilization system for SRS treatments of brain tumors.

Based on the results of treatment and a positive outcome in most patients treated with stereotactic radiotherapy, treatment of patients with multiple brain metastases has begun.

Several patients with multiple intracranial metastases were irradiated during the same day (Figure 5).

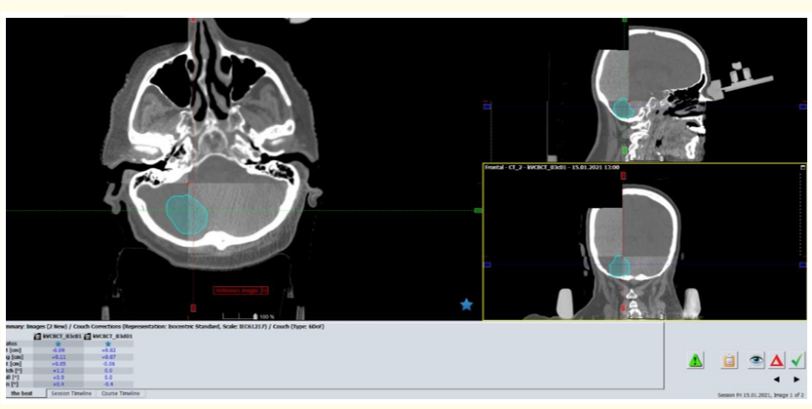

Figure 4: Comparison of deviations of pretreatment and posttreatment verification by CBCT imaging.

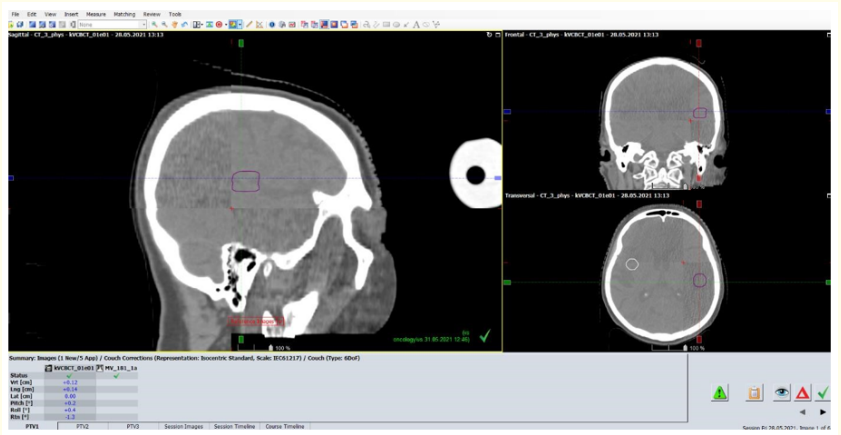

Figure 5: Pretreatment CBCT of a patient with multiple metastatic disease.

Patients were treated with SRS technique with multiple metastases, a total of 5 metastatic changes during the same day. The total dose planned for each individual lesion is 12 Gy. Each metastatic lesion had a special plan with a defined treatment isocenter.

Patients tolerated the outcome of the treatment well, with no acute adverse reactions.

Geometric deviation on the first fraction of setup patients were measured in total of 35 patients according to pretreatment CBCT imaging verification. Deviations in translational and rotational movements shown on pre-treatment CBCT images were calculated (Chart 4). 


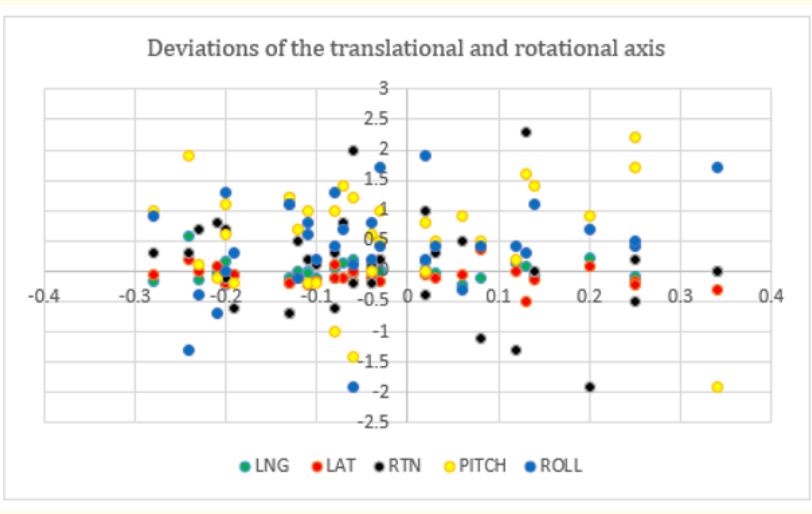

Chart 4: Deviation range in translational and rotational movements calculated based on CBCB imaging.

Deviations in vertical movement from $-0.28 \mathrm{~mm}$ to $0.31 \mathrm{~mm}$ in range with a mean value in the amount of $-0.03 \mathrm{~mm}$ of all 35 measurements. Deviations in longitudinal movement from $-0.22 \mathrm{~mm}$ to $0.23 \mathrm{~mm}$ in range with the mean value of $0.004 \mathrm{~mm}$.

Deviations in lateral movement from $-0.23 \mathrm{~mm}$ to $0.33 \mathrm{~mm}$ in range with a mean value of $-0.07 \mathrm{~mm}$. The results showed that the deviations were minimal, in most cases submilimeter and proved the accuracy of positioning the patient with the specified immobilization equipment.

According to the results so far, in the first patients irradiated with the stereotactic technique, good therapeutic effects of local disease control were achieved in terms of complete eradication and reduction of metastatic changes. Longer follow-up is required to assess late toxicity and final clinical outcome and eventual recurrence of the disease.

\section{Materials and Methods}

In this research article, retrospectively analyzed and were included a total of 35 patients with brain tumors treated with stereotactic radiotherapy in the period from 2019 to 2021. Data were taken from the oncology information system Aria, (Varian, Palo Alto, CA). All the patients tretated on linear accelerator True Beam, Varin (Palo Alto, CA). Measurements were performed based on pretreatment images of CBCT on the first fraction of treatment, maximum and mean deviation values were measured.

\section{Results and Discussion}

Since stereotactic radiotherapy is a technique that requires extreme precision in the delivery of treatment, among other things, a treatment delivery device and special equipment for immobilizing the patient are required. Most of the patinets were with metastasies in the brain (28pt) and SRS in a single-fraction treatment regimen (15pt) with the most commonly delivered dose of $18 \mathrm{~Gy}$.

According to the results of the study, the deviations in the first fraction of treatment based on CBCT images were in vertical movement from $-0.28 \mathrm{~mm}$ to $0.31 \mathrm{~mm}$ in range with a mean value in the amount of $-0.03 \mathrm{~mm}$. In longitudinal movement from $-0.22 \mathrm{~mm}$ to $0.23 \mathrm{~mm}$ in range with the mean value of $0.004 \mathrm{~mm}$ and deviations in lateral movement from $-0.23 \mathrm{~mm}$ to $0.33 \mathrm{~mm}$ in range with a mean value of $-0.07 \mathrm{~mm}$. In most of patients deviations were submilimeter and proved the accuracy of positioning the patient with the specified immobilization equipment. Control evaluation of first patients and these resaults, proved SRS to be an effective treatment, which enabled its application for the treatment of multiple brain metastases

\section{Conclusion}

Based on follow-up and evaluation of MRI controls at least 6 weeks after treatment, in patients treated with stereotactic radiation technique, a significant effect of therapy with a good outcome in eradication of metastatic and benign changes in the head region was determined. For multiple metastases, advantages compared with whole brain irradiation include improved local control, fewer neurocognitive side effects, and a shorter treatment course. The application of a linear accelerator with Kv CBCT imaging system and special equipment for patient immobilization for SRS treatment, enables accurate and precise delivery of the treatment with the final good treatment outcome.

\section{Conflict of Interest}

There is no financial interest or conflict of interest.

\section{Bibliography}

1. Kim Y-J., et al. "Single-Dose Versus Fractionated Stereotactic Radiotherapy for Brain Metastases". International Journal of Radiation Oncology*Biology*Physics 81.2 (2011): 483-489.

2. Dumont Lecomte D., et al. "Hypofractionated stereotactic radiotherapy for challenging brain metastases using 36 Gy in six fractions". Cancer/Radiothérapie 23.8 (2019): 860-866. 
3. https://www.mayoclinic.org/tests-procedures/stereotacticradiosurgery/about/pac-20384526

4. Halasz LM and Rockhill JK. "Stereotactic radiosurgery and stereotactic radiotherapy for brain metastases". Surgical Neurology International 4 (2013):

5. Sanad MH., et al. "Radiocomplexation and bioevaluation of 99mTc nitrido-piracetam as a model for brain imaging". Radiochimica Acta 105 (2017): 729.

6. Sanad MH and Alhussein AI. "Preparation and biological evaluation of $99 \mathrm{mTc} \mathrm{N}$ histamine as a model for brain imaging: in silico study and preclinical evaluation". Radiochimica Acta 106 (2018): 229.

7. Amin AM., et al. "Radiochemical and biological characterization of 99mTc-piracetam for brain imaging". Radiochemistry 55 (2013): 624 .

8. Sanad MH., et al. "99mTc-Oxiracetam as a potential agent for diagnostic imaging of brain: labeling, characterization, and biological evaluation". Radiochemistry 60 (2018): 58.

9. Sanad MH., et al. "Radioiodination and bioevaluation of rolipram as a tracer for brain imaging: in silico study, molecular modeling and gamma scintigraphy". Journal of Labelled Compounds and Radiopharmaceuticals 61 (2018): 501.

10. Zhang J., et al. "Synthesis and biodistribution of the $99 \mathrm{mTc}$ (CO)3-DEDT complex as a potential new radiopharmaceutical for brain imaging". Journal of Radioanalytical and Nuclear Chemistry 272 (2007): 91.

11. Satpati D., et al. "Preparation and bioevaluation of 99mTccarbonyl complex of 5-hydroxy tryptamine derivative". Applied Radiation and Isotopes 64 (2006): 888.

12. Erfani M., et al. "Synthesis and biological evaluation of $99 \mathrm{mTc}$ (CO)3-OH-PP-CS2 for brain receptor imaging". Iranian Journal of Nuclear Medicine 20 (2012): 25.

Volume 5 Issue 9 September 2021

(C) All rights are reserved by Enis Tinjak, et al. 